\title{
Research on harmonic analysis and Simulation of grid connected synchronous motor
}

\author{
Jian Huang ${ }^{1, a}$, Bingyang Luo ${ }^{2, b}$ \\ ${ }^{1}$ School of Jian Huang, Wuhan University of Technology, Wuhan 430070, China; \\ ${ }^{2}$ School of Bingyang Luo, Wuhan University of Technology, Wuhan 430070, China. \\ a1092371836@qq.com, b442493007@qq.com
}

Keywords: harmonic, power distribution system, synchronous motor power generation, THD.

\begin{abstract}
Harmonics in power system bring many adverse effects to the distribution system. Based on the analysis of the reason of harmonic generation, this paper puts forward the calculation method of harmonic analysis in the simplified circuit model, establishes a synchronous motor power grid model, simulates in Simulink and gives the main harmonic and total harmonic current distortion rate in the synchronous motor power grid.
\end{abstract}

\section{Introduction}

Ideally, the power supply should provide standard sinusoidal voltage signal for each client, yet the so-called waveform distortion always exists in the actual voltage and current waveform, which means the harmonic distortion.

The harmonic distortion of the power system is getting more serious with the increasing of non-linear loads in the industry, the waveform distortion of voltage or current signal in the grid will create a lot of problems, for instance, transformer overheating, neutral line overload, fuse and electronic relay malfunction and telephone interference. With the development of power electronics technology and HVDC transmission technology, the harm of harmonics caused by the various power electronic equipment has become more and more serious. Whereupon, harmonic research plays a more and more important role in ensuring the reliable operation of the power grid and equipment and predicting the potential problems.

With the rapid development of power electronic technology and computer technology, harmonic generation will not only reduce the power quality, shorten the service life of the equipment, may also cause the maloperation of relay protection device, and has become an obstacle to the development of power electronic technology itself. Meanwhile, people now pay more attention to the green power system. Thus the research on the reason of harmonic generation and the harm of the harmonic generation is of great significance.

\section{Harmonic sources}

Many industrial, commercial, and residential facilities will cause waveform distortion. Different nonlinear loads will produce different but discernible harmonic spectrum, which makes the task of identifying harmonic distortion of the initiator becomes feasible. We must give proper consideration to the peak value of parallel resonance in the process of evaluating waveform distortion, otherwise, if we use a single tuned filter to eliminate a characteristic harmonic in some position, the shunt peaking (pole) of the filter will just be consistent with a low order characteristic harmonic of a load, then the filter will not eliminate filter, but amplify waveform distortion. On the other hand, the power quality of the public power supply will be reduced by the heavy harmonic load on the weak feeder.

\subsection{Traditional harmonic sources}

Variable electronic stations which work in saturated zone and user transformer act as the main harmonic sources before the popularization of power electronic switch equipment. Nowadays, many cases would lead to harmonic distortion, transformer saturation when peak demand state and load 
state of the small step-up process are just two kinds of situations. Power converter and electric arc furnace are the main harmonic sources in the industrial application of high power, if harmonic frequency generated by the arc device is lower than the fundamental frequency, so called subharmonic; if harmonic frequencies are not integer times than the fundamental frequency, so called interharmonics.

\subsection{Prospective harmonic sources}

Harmonic sources of electrical system in the future will be more diverse, and sensitive electronic devices are constantly used, as a result of which, the new system designed in the future should be able to run in the environment in which the harmonic level is increasing.

\section{Influences of harmonic on power distribution system}

Residential users lack of awareness of harmonics caused by household appliances, even the industrial and commercial users don't know the harmonic filter very well, which led to many power users suffering from adverse effects caused by harmonic distortion to various loads. In a broad sense, these adverse effects will be discussed in the following chapter.

\subsection{Thermal effect of transformer}

Modern industrial and commercial networks are deeply influenced by the large number of harmonic currents generated by various nonlinear loads, such as variable speed drives, arc furnaces and fluorescent lamps. All of the devices described above need to be supplied with power through the transformer. Transformer with delta-star connection or delta-delta connection should have a zero-sequence current, otherwise it will lead to overheating of the neutral line. The circulation in the triangular connection causes the current to become larger and generates additional heat. We should pay attention to this problem. The zero-sequence current can not be reflected by the current in the high voltage side of the transformer with triangular connection, but the heat loss caused by them can not be ignored.

\subsection{Abnormal action of electronic relays}

In practical applications, it is often encountered that the frequency conversion driving equipment action leads to shutdown, and the harmonic distortion should be assessed. Although the unexpected overload is the main reason for the abnormal action of the relay protection, the serious harmonic distortion caused by the increase of the nonlinear load can also cause the abnormal action of the relay protection.

On the other hand, in the application of the frequency conversion device, the 3rd harmonic currents caused by the serious imbalance of the line current may also cause the fault trip of relay protection. Therefore, it is necessary to evaluate the abnormal action of the relay protection in the device with nonlinear load. The specific method is to detect the harmonic distortion level, and check whether the relay needs to be fine tuned. If there is an abnormal action of relay protection in industrial facilities, we should begin to consider the filtering of harmonics.

\subsection{Lighting equipment}

The harmonic whose frequency is not an integer multiple of the fundamental frequency is called inter-harmonic, inter-harmonic can make the voltage oscillation, which led to the lights flashing. The inter-harmonics are mainly produced by cycloconverters which are widely used in iron and steel, cement, mining and other industrial and arc welding machine and arc furnace. According to the TF IEEE and WG CIGRE/CIRED joint standard of the inter-harmonic, the characteristic frequency of cycloconverter is

$$
f_{i}=\left(p_{1} \cdot m \pm 1\right) f_{1} \pm p_{2} \cdot n \cdot f_{0}
$$

In the formula, $f_{i}$ is the frequency of inter-harmonic; $f_{1}$ is the frequency of fundamental frequency; $p_{1}$ and $p_{2}$ are pulse numbers of ballast and output area respectively; $m$ and $n$ are integers like $0,1,2,3$ and so on, but they cannot be 0 at the same time; $f_{0}$ is output frequency of the cycloconverter.

Superposition between the constant-frequency multi harmonic voltage and the fundamental wave 
voltage causes the lights flicker, which becomes the main adverse effects of inter-harmonics.

\subsection{Problems to consider in the operation of cables and equipment in harmonic environment}

With the increasing degree of harmonic distortion in power system, we need to re consider the selection criteria of power equipment in industrial facilities. For example, when the distributed generator works in a nonlinear load dominated environment, the traditional generator designed with higher order instantaneous reactance usually can cause serious thermal effects and eventually make the equipment failure. Therefore, we must limit the operating parameters of the devices, so that these devices can withstand the adverse effects brought by the harmonic in the worst case. Of course, the situation is much more complex than that of the device and network components that are considered in the original state.

\section{Harmonic analysis}

\subsection{Simplified calculation}

When the system is shown alike in Fig.1 circuit description, it can use the simplified manual algorithm. In some important results of the circuit, we can get the resonant frequency of the system by using the following expression:

$$
f_{s}=\sqrt{\frac{S_{c}}{Q_{c}}}=\sqrt{\frac{X_{c}}{X_{l}}}
$$

In the formula, $f_{s}$ is the resonant frequency of the system, and is usually the integer multiples of the fundamental frequency; $S_{c}$ is Short Circuit MVA power of the system on considered resonant point; $Q_{c}$ is capacitors' rated MVA power of system voltage; $X_{c}$ is capacitors' reactance of fundamental frequency; $X_{l}$ is the short-circuit reactance of power supply substation.

If calculated resonant frequency and the harmonic source loads (nonlinear) of a characteristic harmonic frequencies are close to each other, then the resulting great harmonic voltage will cause serious distortion. This requires more accurate analysis and in-depth study.

After simplification, the system impedance in the frequency domain can be approximately expressed as:

$Z(\omega)=\frac{(R+j \omega L)}{\left[1-\omega^{2}(L C)+j \omega R C\right]}$

In the formula, the system impedance $Z(\omega)$ is a function of frequency $(\omega=2 \pi f)$.

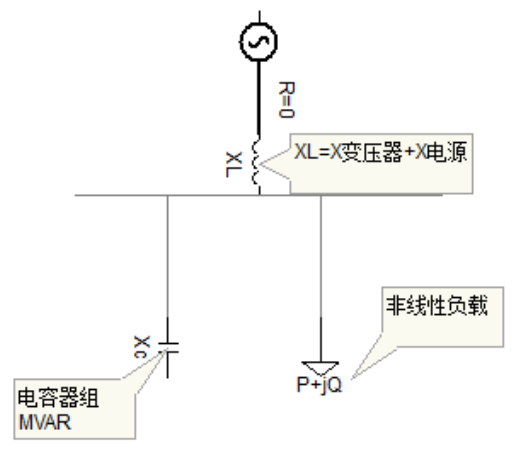

Fig.1 Simplified circuit

Thus, the harmonic voltage of some harmonic frequency can be obtained as follows:

$V_{h}=I_{h} Z_{h}$

Taking all orders of harmonics into account, the effective value of voltage can be obtained as:

$V_{R M S}=\sqrt{V_{1}^{2}+\sum_{h=2}^{n} V_{h}^{2}}$ 
We can estimate total harmonic distortion rate THD and telephone interference factor TIF:

$$
\begin{gathered}
\text { THD }_{V}=\frac{\sqrt{\sum_{h=2}^{\infty} V_{h}^{2}}}{V_{1}} \\
\text { THD }_{I}=\frac{\sqrt{\sum_{h=2}^{\infty} I_{h}^{2}}}{I_{1}} \\
T I F=\frac{\sqrt{\sum_{h=2}^{\infty} \omega_{h}^{2} I_{h}^{2}}}{I_{R M S}}
\end{gathered}
$$

In the formula, the definition of the index is defined by the concept of the total harmonic distortion rate with appropriate weight coefficients $\omega_{h}$, the weight coefficient $\omega_{h}$ reflects the sensitivity of the human ear to the noise of different frequencies. More accurate results can be obtained if the single harmonic voltage and current are used.

\subsection{Analysis example}

If the rotor speed $n$ of the three-phase AC motor and the frequency of the stator current $f$ meet the equation of the relationship $n=\frac{60 f}{p}$, the motor is known as the synchronous motor.

When synchronous generators are connected into parallel or grid, in order to avoid the large impact current with power grid, synchronous generators connected into grid should meet three conditions, namely same phase and frequency with grid, and the excitation EMF and grid voltage are equal in size and direction.

To achieve grid-connected, the first condition must be met, the other two may allow a slight discrepancy. According to the specific requirements of grid-connected: In order to ensure safety, the system voltage deviation is not more than $10 \%$ of the rated voltage, the phase deviation is not more than 10 degrees, the frequency deviation is not more than $1 \%$.

This paper gives the model of synchronous motor power generation and power generation, as shown in Fig.2.

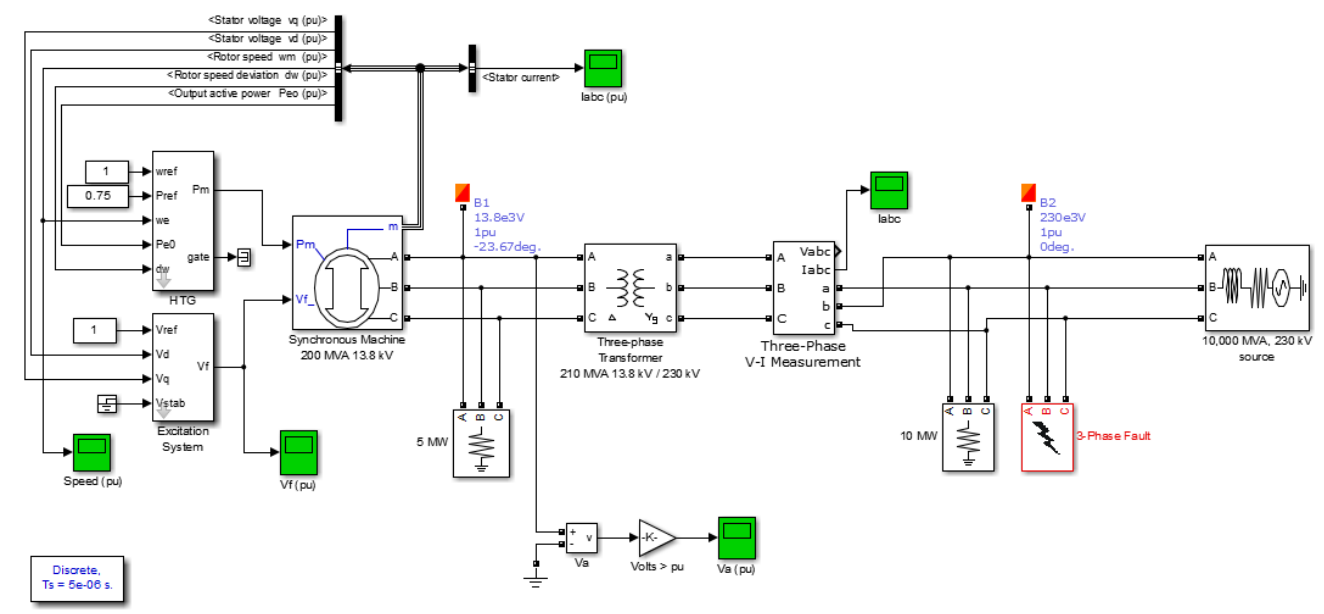

Fig.2 Model of grid-connected synchronous motor

The power supply of the synchronous motor is supplied via the transformer. After the B2 position is connected to the ground fault, the current waveform and harmonic spectrum of the simulation are shown in Fig.3 and Fig.4. 

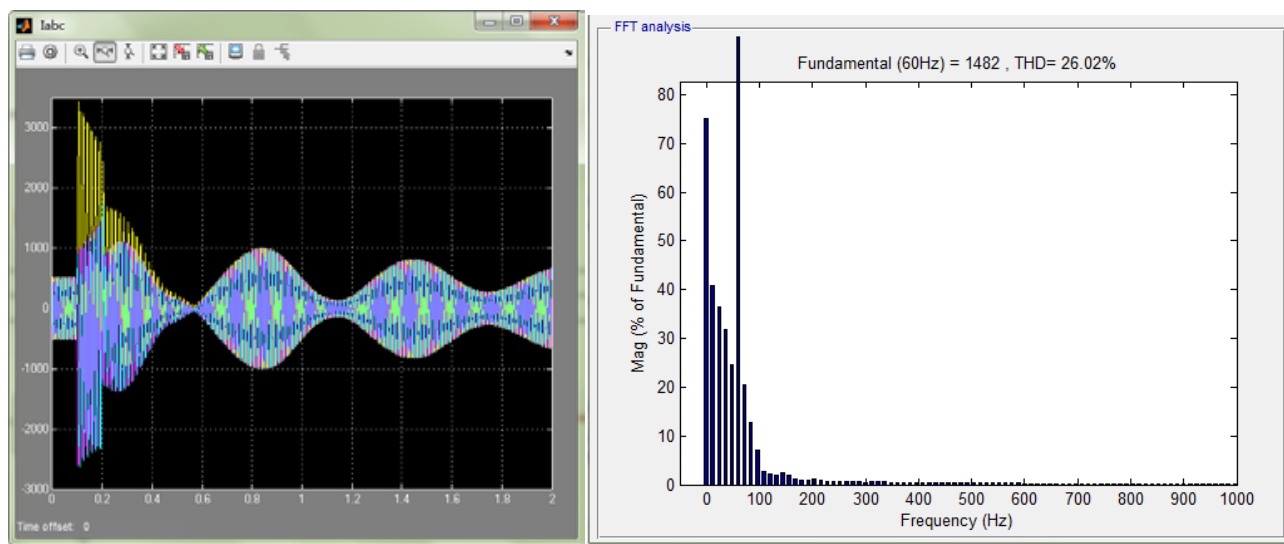

Fig.3 Three phase current waveform during B2 position grounding fault

Fig.4 The current harmonic spectrum of B2 position after the coincidence gate

It can be seen that there are mainly 2nd, 3rd and 5th harmonic currents in the $60 \mathrm{~Hz}$ current, and the total harmonic distortion rate of the $\mathrm{B} 2$ position is $26.02 \%$.

\section{Summary}

This paper analyzes the harm and the main harmonic sources, and the harmonics of power generation and grid-connected are analyzed in view of the problem that the distributed generator can generate large harmonics in the environment with nonlinear load. A simulation model of synchronous generator is established in MATLAB, and the main harmonic and current total harmonic distortion rate are achieved through the analysis of the current harmonic spectrum and the FFT analysis window, which provide the theoretical data for harmonic suppression in the future.

\section{References}

[1]. Jia Wen. Harmonic detection and analysis of three phase bridge circuit [J]. Shanxi Electronic Technology, 2014,04:18-20.

[2]. Yongpei Chen. Application and development of power harmonic in electric power measurement [J]. Heilongjiang Science and Technology Information, 2014,10:37.

[3]. Shan Chi. Harm and suppression of harmonics in electrical system design of civil buildings [J]. Electrical applications, 2013,06:66-71.

[4]. Dajun Tao, Baojun Ge. The harmonic characteristics of the starting low frequency operation stage of the static frequency converter of the power generation motor [J]. Chinese Journal of Electrical Engineering, 2014,36:6450-6457.

[5]. Qing Zhong. Design of synchronous generator in parallel connection training [J]. Modern enterprise education, 2012,22:257-258.

[6]. Hong Liu. Distributed grid connected resonant suppression strategy based on grid connected voltage control [J]. Power grid technology, 2015,02:334-340.

[7]. Zhibin Ye. The harm and control of current harmonic to electrical equipment [J]. Technology and enterprise, 2011,16:82.

[8]. Xiyang Song, Zhong Zhang. Harm and control of power system harmonics [J]. Electrical technology of intelligent buildings, 2009,05:63-66. 